\title{
Características inmunofenotípicas y clínicas del carcinoma renal mucinoso tubular y de células fusiformes
}

\author{
Arias LF*,***, Blanco J*, Hernández S*, Bocardo G**, González L*. \\ *Departamento de Anatomía Patológica. **Departamento de Urología, Hospital Clínico San Carlos, Madrid. \\ ***Afiliación actual: Departamento de Patología, Facultad de Medicina, Universidad de Antioquia, \\ Medellín, Colombia.
}

Actas Urol Esp. 2206;30(7):649-654

\begin{abstract}
RESUMEN
CARACTERÍSTICAS INMUNOFENOTÍPICAS Y CLÍNICAS DEL CARCINOMA RENAL MUCINOSO TUBULAR Y DE CÉLULAS FUSIFORMES

Introducción: $\mathrm{El}$ carcinoma renal mucinoso y tubular de células fusiformes es una neoplasia recientemente incluida en la clasificación de tumores renales. Aún desconocemos aspectos moleculares y de su evolución. Nuestros objetivos son describir características inmunofenotípicas del tumor y aportar casos adicionales para un mejor conocimiento de la neoplasia.

Material y métodos: Realizamos inmunohistoquímica con un amplio panel de anticuerpos a 4 tumores $(0,89 \%$ de carcinomas renales en nuestro centro). Correlacionamos con hallazgos clínicos y tratamos de determinar patrones de inmunotinción.

Resultados: Todos los casos marcaron intensa y difusamente para citoqueratina (CK)7. La inmunotinción fue variable para antígeno de membrana epitelial, vimentina, S-100, antígeno Ulex europaeus, CK de alto peso molecular, CK8, CK18 y CK19. Los tumores fueron homogéneamente negativos para antígeno carcinoembriónico, CD15, CD10, CD34, desmina, actina, CK10, CK20 y HMB45. El indice proliferativo (Ki67) fue menor del 1\% en todos los casos. La marcación fue similar en células cúbicas y fusiformes.

Conclusiones: Este tumor presenta un inmunofenotipo variable de acuerdo con diferentes trabajos y no se conoce con precisión su histogénesis o la célula hacia la cual se diferencian. La similitud en la expresión de marcadores en células cúbicas y fusiformes confirma un mismo origen y diferenciación.
\end{abstract}

Palabras clave: Mucinoso. Células fusiformes. Carcinoma de células renales. Riñón. Neoplasias. Inmunohistoquímica.

\section{ABSTRACT \\ IMMUNOHISTOCHEMICAL PROFILE AND CLINICAL FEATURES OF MUCINOUS TUBULAR AND SPINDLE RENAL CELL CARCINOMA}

Introduction: We present the immunohistochemical (IHC) analyses of a series of four kidney tumors currently classified as mucinous tubular and spindle renal cell carcinoma (WHO), a tumor with uncertain histogenesis and differentiation. Our aims were to determine an immunoprofile and to add clinical and morphological information about this rare renal carcinoma.

Material and methods: The four tumors were found between 415 renal carcinomas at our center (0.89\%). IHC was carried out with a panel of antibodies in these four cases. Morphogical and clinical information was collected and analyzed.

Results: All tumors were well circumscribed and confined to the kidney. Immunoreactivity for cytokeratin (CK)7 was intense and diffuse. Immunostaining was variable for EMA, vimentin, S-100, Ulex europaeus antigen, high-molecular-weight CK, CK 8, CK18, and CK 19. The tumors were homogeneously negative for CEA, CD15, CD10, CD34, desmin, actin, CK10, CK20, and HMB45. Proliferative index (Ki67) was <1\% en all cases. The immunoreactivity was similar in cuboidal and spindle cells.

Conclusions: The present tumor shows a variable immunophenotype and there is not a precise cell type differentiation. The immunostaining in cuboidal and spindle cells suggests a similar differentiation and histogenesis.

Keywords: Mucinous. Spindle cell. Renal cell carcinoma. Kidney. Neoplasms. Immunohistochemistry. 
$\mathrm{R}$ ecientes avances en la caracterización morfoógica y citogenética de las neoplasias renales epiteliales en adultos han llevado a la formulación de una nueva clasificación de estos tumores. Esta clasificación está basada en la morfología, pero refleja las características genéticas de las diferentes categorías de neoplasias ${ }^{1}$. No obstante, es claro que aún queda mucho por conocer de las lesiones neoplásicas renales. En la clasificación más reciente se incluye una neoplasia epitelial con una morfología muy característica: estroma mixoide, disposición de las células formando estructuras tubulares y cordones, áreas fusocelulares, citología de bajo grado y bordes poco infiltrativos ${ }^{1}$. En la nueva clasificación este tumor es llamado: "carcinoma mucinoso, tubular y de células fusiformes" ("mucinous tubular and spindle cell carcinoma"). Otros nombres que se han utilizado en años anteriores para esta neoplasia han sido: "neoplasia renal epitelial mixoide de bajo grado" ("low-grade myxoid renal epithelial neoplasm") 2 , "carcinoma renal de células cúbicas y fusiformes" ("spindle and cuboidal renal cell carcinoma") ${ }^{3}$, y "carcinoma renal fusocelular de bajo grado" ("Low-grade spindle cell carcinoma of the kidney") 4 . La neoplasia corresponde a menos del 1\% de carcinomas renales. En esta lesión la expresión de diversos marcadores moleculares ha sido variable en distintos trabajos y algunos autores han propuesto que se clasifique de acuerdo a la expresión de antígenos por inmunohistoquímica (IHQ), sugiriendo que debe expresar citoqueratina $(\mathrm{CK})$ de alto peso molecular y ser negativa para CD15² sin embargo, otros trabajos demuestran que algunos o muchos de estos tumores no expresan CK de alto peso y otros son positivos para CD15 $5^{5,6}$

Nosotros estudiamos cuatro casos de esta lesión que en la actualidad es bien reconocida. Nuestros objetivos son: evaluar su perfil de expresión de marcadores de IHQ caracterizando patrones de inmunotinción y tratar de que todos conozcamos su morfología, presentación clínica y evolución.

\section{MATERIAL Y MÉTODOS}

Revisamos 451 carcinomas renales resecados en el Hospital Clínico San Carlos de Madrid entre 1984 y 2004 y encontramos cuatro casos con la morfología de carcinoma mucinoso tubular y de células fusiformes $(0,89 \%)$. Todos los cristales con cortes histológicos de la lesión fueron revisados y seleccionamos bloques de tejido incluido en parafina para hacer IHQ en fragmentos de tumor con áreas tubulares y áreas fusocelulares.

\section{Inmunohistoquímica}

Para la inmunomarcación, cortes de $4 \mathrm{~mm}$ de espesor fueron desparafinados en xilol por 30 minutos y rehidratados usando diferentes concentraciones de etanol. Los anticuerpos utilizados, fabricantes, diluciones y sistema de recuperación antigénica aparecen en la Tabla 1 . Realizamos la IHQ utilizando el procedimiento de marcación con peroxidasa, el sistema EnVision $+{ }^{\circledR}$ (Dako ${ }^{\circledR}$, Glostrup, Dinamarca) y diaminobenzidina como cromógeno, con el sistema automatizado de tinción Biotek-1000 (Biotek Solutions, Inc. ${ }^{\circledR}$, Tucson, AZ, USA)

Simultáneamente, utilizamos controles positivos para todos los marcadores. Los resultados fueron evaluados como positivos si la inmunotinción se detectó en más del 5\% de las células tumorales en la membrana citoplasmática (CD10), en el citoplasma y el núcleo (S-100) o en el citoplasma y/o la membrana celular (todos los otros anticuerpos). La extensión de la tinción fue graduada como: $1+$ (tinción en $>5 \%$ y $\leq 30 \%$ de células tumorales); $2+$ (tinción en $>30 \% \leq 50 \%$ ), y $3+$ (tinción en $>50 \%$ ). Determinamos el índice de proliferación celular utilizando IHQ para Ki67; el indice fue expresado como porcentaje de células con tinción nuclear.

\section{RESULTADOS}

Las características clínicas aparecen resumidas en la Tabla 2. En todos los pacientes se realizó nefrectomía sin otra terapia adicional. La media del período de seguimiento fue de 46,3 meses (rango: 3 - 106 meses); en ninguno de los casos se ha detectado recurrencia o metástasis.

\section{Características histopatológicas}

Todos los tumores tenían aspecto sólido, estaban bien circunscritos y en ninguno de ellos se encontró extensión extrarrenal. El diámetro ma- 
Tabla 1

Anticuerpos primarios utilizados para inmunohistoquímica

\begin{tabular}{|c|c|c|c|c|}
\hline Anticuerpo & Clon & Dilución & Fabricante & Pretratamiento \\
\hline EMA & $\mathrm{E}-29$ & $1: 300$ & DAKO, Glostrup, Dinamarca & Vapor - bufer citrato \\
\hline CEA & II7 & $1: 1000$ & DAKO, Glostrup, Dinamarca & Vapor \\
\hline VIM & Vim3B4 & $1: 500$ & Novocastra, Newcastle, U.K. & Vapor \\
\hline S-100 & policlonal & $1: 1000$ & DAKO, Glostrup, Dinamarca & Vapor \\
\hline Ulex & policlonal & $1: 100$ & DAKO, Glostrup, Dinamarca & Vapor \\
\hline Desmina & DE-R-11 & $1: 200$ & DAKO, Glostrup, Dinamarca & Vapor \\
\hline Actina & HHF35 & $1: 50$ & DAKO, Glostrup, Dinamarca & Vapor \\
\hline CD 10 & $56 C G$ & $1: 20$ & Novocastra, Newcastle, U.K. & EDTA y Vapor \\
\hline CD 15 & C3D-1 & $1: 25$ & DAKO, Glostrup, Dinamarca & Vapor \\
\hline CD 34 & QBEnd10 & $1: 100$ & DAKO, Glostrup, Dinamarca & Vapor \\
\hline HMB 45 & HMB 45 & $1: 50$ & DAKO, Glostrup, Dinamarca & Vapor \\
\hline HMW-CK & $34 ? \mathrm{E} 12$ & $1: 25$ & DAKO, Glostrup, Dinamarca & Vapor \\
\hline CK 7 & OV-TL12/30 & $1: 100$ & DAKO, Glostrup, Dinamarca & EDTA y Vapor \\
\hline CK 8 & TS1 & $1: 40$ & Novocastra, Newcastle, U.K. & Vapor \\
\hline CK 10 & LHP1 & prediluido & Novocastra, Newcastle, U.K. & Tripsina \\
\hline CK 18 & DC-10 & $1: 40$ & Novocastra, Newcastle, U.K. & Vapor \\
\hline CK 19 & b- 170 & $1: 150$ & Novocastra, Newcastle, U.K. & Tripsina \\
\hline CK 20 & $\mathrm{~K}_{\mathrm{S}} 20.8$ & $1: 50$ & Novocastra, Newcastle, U.K. & Vapor \\
\hline Ki67 & KiS5 & $1: 10$ & DAKO, Glostrup, Dinamarca & EDTA y Vapor \\
\hline
\end{tabular}

EMA: antígeno epitelial de membrana; CEA: antígeno carcinoembriónico; VIM: vimentina; CK: citoqueratina; HMW-CK: CK de alto peso molecular; EDTA: ácido etilendiaminotetraacético.

Tabla 2

Características clínicas

\begin{tabular}{cccccc}
\hline Caso & Edad/sexo & Tamaño $(\mathbf{c m})$ & Presentación clínica & Estadio UICC & Seguimiento \\
\hline 1 & $52 / \mathrm{F}$ & 3,8 & Dolor abdominal & I & NR \\
2 & $62 / \mathrm{F}$ & 11,0 & Dolor abdominal & III & NR \\
3 & $39 / \mathrm{M}$ & 4,3 & Hallazgo incidental & I & NR \\
4 & $51 / \mathrm{F}$ & 6,0 & Hematuria & $\mathrm{I}$ & $\mathrm{NR}$ \\
\hline
\end{tabular}

UICC: Unión Internacional Contra el Cáncer; NR: no recurrencia

yor de los cuatro tumores fue de $3,8 \mathrm{~cm}, 4,3 \mathrm{~cm}$., $6,0 \mathrm{~cm}$ y $11,0 \mathrm{~cm}$ respectivamente; en ninguno de ellos pudo determinarse una localización cortical o medular del epicentro del tumor, ya que comprometían ambas porciones del parénquima renal. No se identificó cápsula rodeando la lesión y ésta comprimía el tejido adyacente sin presentar bordes infiltrativos. Microscópicamente los tumores están formados por células cúbicas de tamaño mediano con citoplasma débilmente eosinófilo, se disponen formando estructuras tubulares bordeadas por una fila de células. Estos túbulos son elongados, rectos, curvos y muchos de ellos se interconectan; otros aparecen con un espacio central delgado, como una hendidura (Fig. 1). En algunas zonas las células tumorales adoptan un patrón más sólido con disposición en láminas. 


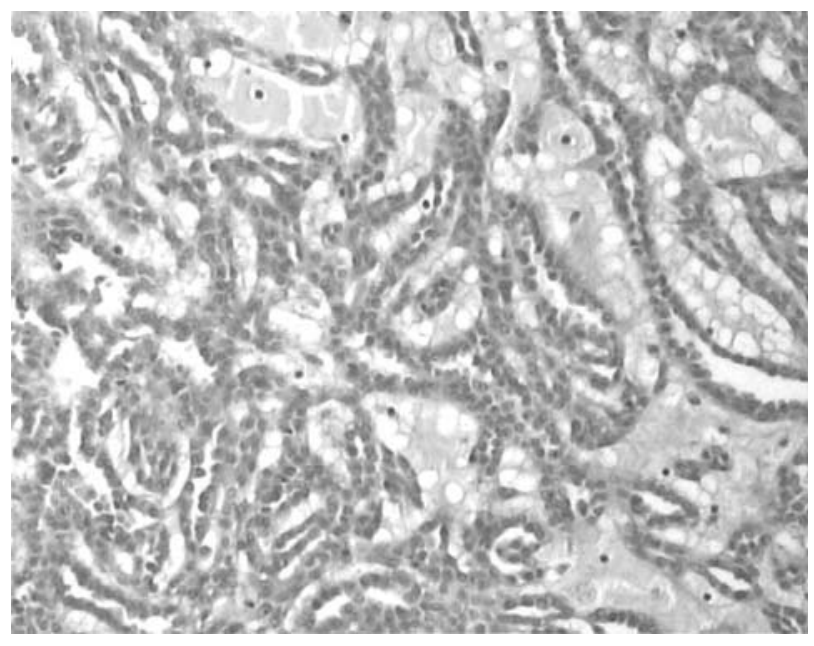

Figura 1. Túbulos bien formados con aspecto elongado, rectos y curvos; los espacios intercelulares tienen aspecto mixoide, mucoide o edematoso. Nótese el poco pleomorfismo celular y la relativa homogeneidad de los núcleos (H\&E, aumento original, X200).

Múltiples áreas tumorales, en una proporción variable, están formadas por células fusiformes, con escaso citoplasma y núcleos elongados, homogéneos, con nucléolos poco notorios. Estas células adoptan un patrón fascicular o pueden verse formando láminas sólidas, estas áreas tienen un aspecto parecido al de leiomiomas (Fig. 2). En los cuatro casos el estroma presenta áreas basófilas, mixoides o con aspecto edematoso. Algunos cordones de células cúbicas parecen flotar en el

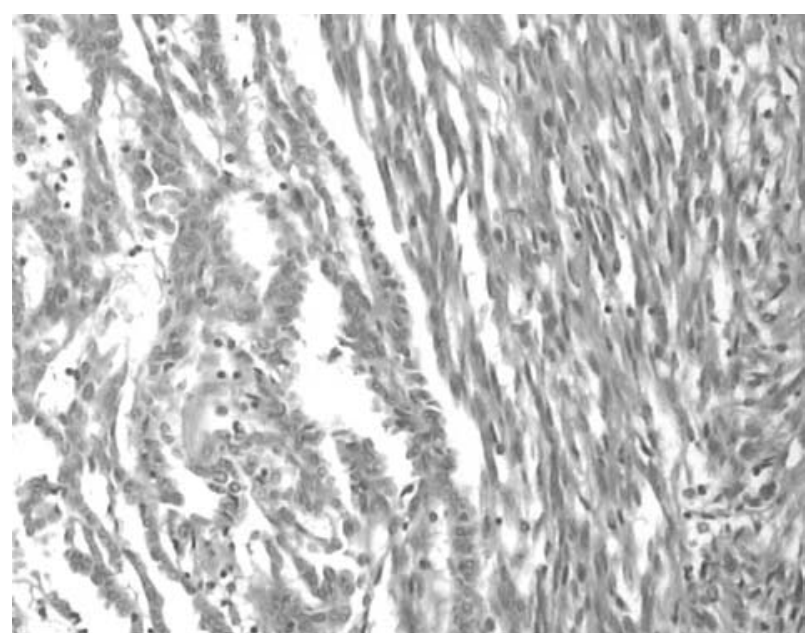

Figura 2. A la izquierda de la microfotografia hay células cúbicas formando estructuras tubulares; a la derecha células fusiformes formando fasciculos, sin atipia nuclear ni otras carcateristicas sarcomatoides. Ambos componentes celulares tienen el mismo inmunofenotipo. (H\&E, aumento original, X200). material mucoide. Hay muy pocas figuras mitóticas, con un rango de 0 a 4/50 campos de gran aumento; no hay mitosis atípicas. En dos casos identificamos necrosis, en uno de ellos fue extensa y en el otro focal. Todas las neoplasias tienen un grado 2 de Fuhrman. Estas características histológicas son similares a las descritas por otros autores.

\section{Hallazgos de inmunohistoquímica}

Todos los casos son completamente negativos para CEA, CD15, CD10, CD34, desmina, actina, HMB45, CK10 y CK20. Los resultados con los otros anticuerpos aparecen en la Tabla 3. Similar a varios trabajos previos, nuestros casos son difusamente reactivos para CK7. La inmunotinción para Ki67 fue menor del 1\% de células tumorales en todos los cuatro casos. La tinción fue similar en células cúbicas y fusiformes. No encontramos un patrón citológico particular de tinción con ninguno de los marcadores.

\section{DISCUSIÓN}

El carcinoma renal mucinoso tubular y de células fusiformes es en la actualidad una lesión bien reconocida ${ }^{7,8}$ de la que aún no sabemos con claridad su célula de origen o hacia que tipo de célula se diferencia. Diferentes trabajos están de acuerdo en que estos tumores tienen un bajo grado de malignidad, bajo grado nuclear, bajo índice de proliferación celular y, usualmente, presentan bordes poco infiltrati$\operatorname{vos}^{6}$. Su comportamiento es relativamente indolente, aunque, se debe tener cautela debido a que en la literatura se han descrito algunos casos de tumores con aspecto histológico similar, antes de la clasificación actual, que han presentado metástasis ${ }^{9}$. En el diagnóstico diferencial es muy importante distinguir este tumor de carcinomas renales con áreas sarcomatoides debido a que estas últimas son neoplasias con alto grado de malignidad; de hecho, ha sido frecuente en el pasado encontrar casos diagnosticados así. En el componente sarcomatoide de carcinomas renales hay células fusiformes pleomórficas, de alto grado, con abundantes mitosis, incluyendo atípicas, y no se identifican las áreas mixoides y arquitectura de túbulos interconectados que se evidencian en la 
Tabla 3

Hallazgos de inmunohistoquímica ${ }^{1}$

\begin{tabular}{lcccc}
\hline Anticuerpo & Caso 1 & Caso 2 & Caso 3 & Caso 4 \\
\hline EMA & negativo & $3+$ & negativo & $3+$ \\
VIM & $3+$ & $1+$ & negativo & $2+$ \\
S-100 & $1+$ & negativo & negativo & negativo \\
Ulex & $3+$ & $1+$ & $1+$ & negativo \\
APM-CK & negativo & $1+$ & negativo & $1+$ \\
CK 7 & $1+$ & $3+$ & $3+$ & $3+$ \\
CK 8 & negativo & $1+$ & $2+$ & negativo \\
CK 18 & negativo & $2+$ & $3+$ & negativo \\
CK 19 & negativo & $2+$ & $1+$ & $2+$ \\
\hline
\end{tabular}

EMA: antígeno de membrana epitelial; VIM, vimentina; CK: citoqueratina; APM-CK: CK de alto peso molecular; negativo: tinción en $<5 \%$ de células tumorales; $1+$ : tinción en $>5 \%$ y $<30 \%$ de células; $2+$ : tinción en $>30 \%$ y $<50 \%$ de células; $3+$ : tinción en $>50 \%$ de células. ${ }^{1}$ No están incluidos anticuerpos negativos en los cuatro casos.

lesión que nos ocupa. De acuerdo con nuestra exhaustiva revisión de la literatura, no se han descrito casos de carcinoma renal mucinoso tubular y de células fusiformes con verdadero cambio sarcomatoide. De otro lado, en dos de nuestros casos encontramos necrosis (extensa en uno y focal en el otro), pero, este hallazgo no parece sugerir una evolución más agresiva de acuerdo a nuestro trabajo y a lo informado por otros autores.

El perfil de IHQ de estos tumores no está completamente claro: los resultados con EMA, CD15, $34 \mathrm{bE} 12$ y S-100 han sido variables ${ }^{2,5,6}$. Es evidente que tumores con características morfológicas similares pueden tener diferente expresión inmunohistoquímica, sin que esto signifique, necesariamente, que son tumores distintos. Muchas de las clasificaciones de tumores de diversos órganos han sido basadas, principalmente, en su morfología; el inmunofenotipo no es siempre exactamente similar entre tumores clasificados como del mismo tipo. En nuestros casos, las características morfológicas y el bajo grado de malignidad son aspectos importantes para clasificar estos tumores como un tipo específico. Algunos autores han propuesto separar neoplasias con la morfología de carcinoma mucinoso tubular y de células fusiformes de acuerdo a la expresión de diferentes marcadores ${ }^{2}$, sin embargo, la expresión de estos antígenos no parece suficiente para separar tumores con similares características microscópicas, presentación clínica y evolución. Hasta que conozcamos análisis citogenéticos precisos de múltiples casos, hasta ahora inexistentes, que nos permitan aclarar el tipo de diferenciación de sus células o posibles implicaciones terapéuticas o de pronóstico, debemos seguir clasificando estos tumores de acuerdo a sus características morfológicas, a la vez que debemos ser concientes de su variabilidad inmunofenotípica.

El origen de las células de esta neoplasia no se conoce; se ha postulado, basados sólo en la morfología y ultraestructura, que se originan en (o se diferencian hacia) células del asa de Henle o del túbulo contorneado distal ${ }^{2,9,10}$, sin embargo, la complejidad de su expresión antigénica no ha permitido probar este origen o esta diferenciación ${ }^{11}$. La negatividad para CD10 y para CD15 sugiere que no hay diferenciación hacia células con fenotipo de la parte proximal de la nefro$\mathrm{na}^{2,12}$, de otro lado, las células son negativas para marcadores asociados con diferenciación distal: CEA y Ulex ${ }^{2,13}$. Complicando aún más la dificultad para determinar su histogenesis, un reciente trabajo encuentra expresión de alfametilacil-CoA racemasa (AMACR), un marcador relacionado con el túbulo contorneado proximal, en más del $90 \%$ de estos tumores ${ }^{5}$. La expresión similar, con los diferentes marcadores, en las células cúbicas y fusiformes sugiere la misma histogénesis y diferenciación en ambos componentes.

En conclusión, nuestros resultados, y los de otros trabajos, muestran un variable inmunofenotipo en los carcinomas renales mucinosos tubulares y de células fusiformes. Ellos son consistentemente positivos para CK7 y, en la mayoría de casos, negativos para CD10 y CD15. Las características morfológicas e inmunofenotípicas carecen de especificidad en estas lesiones para determinar un origen o tipo de diferenciación celular. El pronóstico favorable de estas lesiones es una razón importante para separarlas de otros carcinomas renales. Para una caracterización completa de estas neoplasias serán necesarios más estudios citogenéticos, de IHQ y más experiencia clinica. 


\section{REFERENCIAS}

1. Eble JN, Sauter G, Epstein JI, Sesterhenn IA (Eds). World Health Organization Classification of Tumours. Pathology and Genetics of Tumours of the Urinary System and Male Genital Organs. Lyon: IARC Press, 2004;10.

2. Parwani AV, Husain AN, Epstein JI, Beckwith JB, Argani P. Low-grade myxoid renal epithelial neoplasms with distal nephron differentiation. Hum Pathol. 2001;32(5):506512 .

3. Hes O, Mora M, Perez-Montiel DM, Suster S, Curik R, Sokol L, et al. Spindle and cuboidal renal cell carcinoma, a tumour having frequent association with nephrolithiasis: report of 11 cases including a case with hybrid conventional renal cell carcinoma/spindle and cuboidal renal cell carcinoma components. Histopathology. 2002;41(6): 549-555.

4. Lloreta J, Corominas JM, Munné A, Dominguez D, Belsa O, Gelabert A, et al. Low-grade spindle cell carcinoma of the kidney. Ultrastruct Pathol. 1998;22(1):83-90.

5. Paner GP, Srigley JR, Radhakrishnan A, Cohen C, Skinnider BF, Tickoo SK, et al. Immunohistochemical Analysis of Mucinous Tubular and Spindle Cell Carcinoma and Papillary Renal Cell Carcinoma of the Kidney: Significant Immunophenotypic Overlap Warrants Diagnostic Caution. Am J Surg Pathol. 2006;30(1):13-19.

6. Srigley JR. Mucinous tubular and Spindle cell carcinoma. En: Eble JN, Sauter G, Epstein JI, Sesterhenn IA, editores. World Health Organization Classification of Tumours. Pathology and Genetics of Tumours of the Urinary System and Male Genital Organs. Lyon, IARC Press, 2004;40.
7. Eble JN. Mucinous tubular and spindle cell carcinoma and post-neuroblastoma carcinoma: newly recognised entities in the renal cell carcinoma family. Pathology. 2003;35 (6):499-504.

8. MacLennan GT, Bostwick DG. Tubulocystic carcinoma, mucinous tubular and spindle cell carcinoma, and other recently described rare renal tumors. Clin Lab Med. 2005; 25(2):393-416.

9. MacLennan G, Farrow GM, Bostwick DG. Low-grade collecting duct carcinoma of the kidney: Report of 13 cases of lowgrade mucinous tubulocystic renal carcinoma of possible collecting duct origin. Urology. 1997;50(5):679-84.

10. Ordonez NG, Mackay B. Renal cell carcinoma with unusual differentiation. Ultrastruct Pathol. 1996;20(1):27-30.

11. Weber A, Srigley J, Moch H Mucinous spindle cell carcinoma of the kidney. A molecular analysis. Pathologe. 2003; 24(6):453-459.

12. Holm-Nielsen P, Pallesen G. Expression of segment-specific antigens in the human nephron and in renal epithelial tumors. APMIS Suppl 1988;4:48-55.

13. Reuter VE. Renal tumors exhibiting granular cytoplasm Semin Diagn Pathol. 1999 May;16(2):135-145.

Dr. L.F. Arias

Departamento de Patología. Facultad de Medicina Universidad de Antioquia,

Carrera 51D, No. 62-29, Medellín, Colombia

E-mail: luisfer_uda@yahoo.com

(Trabajo recibido el 28 de febrero 2006) 